TI 2013-062/III

Tinbergen Institute Discussion Paper
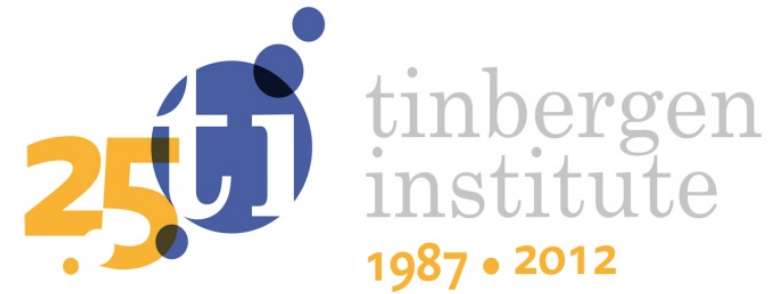

\title{
Solution-Driven Specification of DSGE Models
}

Francisco Blasques

Faculty of Economics and Business Administration, VU University Amsterdam, and Tinbergen Institute. 
Tinbergen Institute is the graduate school and research institute in economics of Erasmus University Rotterdam, the University of Amsterdam and VU University Amsterdam.

More TI discussion papers can be downloaded at http://www.tinbergen.nl

Tinbergen Institute has two locations:

Tinbergen Institute Amsterdam

Gustav Mahlerplein 117

1082 MS Amsterdam

The Netherlands

Tel.: +31(0)205251600

Tinbergen Institute Rotterdam

Burg. Oudlaan 50

3062 PA Rotterdam

The Netherlands

Tel.: +31(0)10 4088900

Fax: $+31(0) 104089031$

Duisenberg school of finance is a collaboration of the Dutch financial sector and universities, with the ambition to support innovative research and offer top quality academic education in core areas of finance.

DSF research papers can be downloaded at: http://www.dsf.nl/

Duisenberg school of finance

Gustav Mahlerplein 117

1082 MS Amsterdam

The Netherlands

Tel.: +31(0)20 5258579 


\title{
Solution-Driven Specification of DSGE MODELS
}

\author{
Francisco Blasques* \\ Department of Finance, VU University Amsterdam \\ Department of Econometrics, VU University Amsterdam \\ Tinbergen Institute
}

April 18, 2013

\begin{abstract}
This paper proposes a functional specification approach for dynamic stochastic general equilibrium (DSGE) models that explores the properties of the solution method used to approximate policy functions. In particular, the solution-driven specification takes the properties of the solution method directly into account when designing the structural model in order to deliver enhanced flexibility and facilitate parameter identification within the structure imposed by the underlying economic theory. A prototypical application reveals the importance of this method in improving the specification of functional nonlinearities that are consistent with economic theory. The solution-driven specification is also shown to have the potential to greatly improve model fit and provide alternative policy recommendations when compared to standard DSGE model designs.
\end{abstract}

Keywords: Nonlinear Model Specification, DSGE, Perturbation Solutions.

JEL Codes: C51, E17, E37.

\section{INTRODUCTION}

Economic theory has always postulated the existence of nonlinear relationships between economic variables. From utility functions that reflect risk aversion to production functions with diminishing returns to scale, the presence of nonlinearities in economic models is pervasive and well established both theoretically and empirically; see e.g. Lau (1986) and Granger and Teräsvirta (1993) for examples of such theoretical and empirical accounts.

\footnotetext{
* A considerable part of this work was developed during the author's visit to Banco de Portugal. The author is thankful to Banco de Portugal for the visiting researcher's grant and the Dutch Science Foundation (NWO) for financial support. The author is also thankful to Andre Lucas, Siem Jan Koopman and those present at the Banco de Portugal Economics Seminar and the VU Amsterdam Internal Econometrics Seminar for helpful comments and suggestions. Corresponding address: VU University Amsterdam, FEWEB/FIN, de Boelelaan 1105, 1081 HV Amsterdam, Netherlands, email: f.blasques@vu.nl.
} 
Unfortunately, the accurate nonlinear design of structural models has often been neglected. This apparent lack of interest for an appropriate nonlinear specification of the model can be traced back to at least five historical factors that exerted a profound influence on the DSGE literature.

First, the most popular techniques for solving DSGE models involved originally the linearization of first-order optimality conditions; see e.g. Blanchard and Kahn (1980). Naturally, this linearization step led the nonlinear specification of functions to take a secondary role. However, in the last three decades, much research has been devoted to developing nonlinear solution methods for DSGE models and today researchers are by no means constrained by linearization requirements; see e.g. Judd (1992, 1998) and Aruoba et al. (2006) for a comparison of methods. Most importantly, in this new world of nonlinear solution methods, the nonlinear specification of the model matters and plays a crucial for the study of phenomena such as time-varying risk premia; see e.g. Uribe (2011).

Second, some lack of concern with functional form misspecification might be explained by the fact that the quantitative analysis of DSGE models has been typically carried out in recognition that the model is misspecified and provides only a stylized description of a potentially very complex data generating process. As a result, misspecification arising only from subtle higher-order nonlinearities might seem comparatively less important than those arising from first order restrictions. As we shall see however, this fact should not reduce our efforts to improve nonlinear functional specification. On the contrary, this paper shows that appropriate modeling of nonlinear dynamics can greatly improve a model's ability to describe fundamental features of the business cycle.

Third, the modeling of nonlinear functional forms has been naturally relegated to second plan by the fact that economic theory provides little guidance about the actual form of utility functions, production functions and others. Indeed, as pointed out by e.g. Lau (1986) and Diewert and Wales (1987) among others, when it comes to nonlinear functional form, economic theory usually suggests only restrictions of a more general nature such as smoothness, monotonicity, concavity, etc. It does not however point to exact parametric specifications. This lack of guidance has often lead to the adoption restrictive parametric functional forms that are justified by the desire for analytical simplicity and algebraic 
tractability. As we shall see, this type of model specification might come however at a very high price, at least, when the researcher is concerned with quantitative analysis, model fit, and the accurate description of business cycle characteristics. The solution-driven specification of the DSGE model avoids these problems by adopting a functional form specification that imposes only those general restrictions captured by the solution method and justified by economic theory.

Forth, paying much attention to nonlinear functional form specification might have seemed rather unnecessary since DSGE models have been often designed to explain only the first and second order moments of the business cycle. As we shall see however, this line of reasoning is an unfounded misconception since low order moments are also functions of parameters determining only high-order nonlinear features of various functions. In particular, the current paper reveals that solution-driven DSGE modeling can greatly improve model fit (by approximately $25 \%$ in the application presented here) even when the notion of "model fit" is based only on matching the auto-covariance structure of the data.

Fifth, despite the existence of a clear theoretical case for the use of nonlinear DSGE models capable of describing phenomena such as time-varying risk premia, the empirical case for nonlinear DSGE models has sometimes been weaker. For example, Fernandez-Villaverde and Rubio-Ramirez (2006) report little gains from the adoption of nonlinear solutions in the description of capital dynamics. ${ }^{1}$ We will see that these results rely however on obtaining higher-order solutions of a model whose functional forms are restrictive and essentially imposed for operational convenience. Hence, any nonlinearities present in the reduced form solution reflect the restrictions imposed from the outset on the DSGE model. In accordance with Fernandez-Villaverde and Rubio-Ramirez (2006), we show here an example where, under a standard DSGE model specification, the nonlinear solutions produce only marginal differences in parameter estimates and model fit, yet great gains are obtained from the same nonlinear solutions when adopting a solution-driven DSGE specification. The importance of nonlinear solutions can thus be greatly enhanced when functional forms are appropriately modeled. In particular, it will become clear that both data and theory call for a functional specification that renders the DSGE model more flexible on those features about which the

\footnotetext{
${ }^{1}$ Empirical support for nonlinear DSGEs has nonetheless been found by several authors; see e.g. Kim and Ruge-Murcia (2009).
} 
theory is silent.

Before moving on, it is also important to note that the solution-driven DSGE specification has a natural place in at least two well-developed strands of the DSGE literature.

First, the solution-driven DSGE specification is founded on the literature devoted to nonlinear solutions of rational expectation models and approximation of policy functions; see e.g. Judd $(1992,1998)$ and Aruoba et al. (2006) for a review. In essence, the solutiondriven DSGE specification ties the DSGE model design to the solution method employed in the policy function approximation.

Second, the solution-driven DSGE specification constitutes a natural extension to the existing literature on DSGE models with flexible functional forms; see e.g. Lau (1986) and Diewert and Wales (1987). The solution-driven specification is however unique in adopting functional forms justified by the nature of the employed solution method.

Section 2 below uses a prototypical DSGE model to introduce the solution-driven DSGE model design. Section 3 describes the main features of the business cycle and explains how these features can be used by an indirect inference estimator to estimate the structural parameters. Finally, Section 4 compares the estimation results obtained form several specifications of the same model. Section 5 concludes.

\section{The Solution-Driven DSGE Specification}

For simplicity, let us consider a prototypical DSGE model similar to that used in Judd (1992). This model postulates that (i) consumption $c_{t}$ is determined by maximizing the expected value of a discounted stream of future utilities defined by an instantaneous utility function $u$; (ii) the capital stock $k_{t}$ is the result of an accumulation process with a fraction $(1-\delta)$ being lost to depreciation and increments determined by investment $i_{t}$ which corresponds to the fraction of output that is not consumed, (iv) output $y_{t}$ is a function $f$ of the capital stock $k_{t}$ and total factor productivity (TFP) shocks $z_{t}$; and finally (v) TFP shocks contain dependence generated by an autoregressive function $g$. This prototypical model is 
thus summarized as,

$$
\begin{aligned}
\max _{\left\{c_{s}\right\}_{s=t}^{\infty}} \mathbb{E}_{t}\left[\sum_{s=t}^{\infty} \beta^{s-t} u\left(c_{s}\right)\right] \\
\text { s.t. } \quad k_{t+1}=(1-\delta) k_{t}+i_{t} \quad, \quad i_{t}=y_{t}-c_{t}, \\
\quad y_{t}=f\left(k_{t}, z_{t}\right) \quad, \quad z_{t}=g\left(z_{t-1}\right)+\epsilon_{t}
\end{aligned}
$$

where $\beta$ is the intertemporal discount rate and the innovations of the TFP process follow an independent Gaussian process $\left\{\epsilon_{t}\right\} \sim \operatorname{NID}\left(0, \sigma_{\epsilon}^{2}\right)$. The first-order optimality conditions for this dynamic optimization problem are well known and given by,

$$
\begin{aligned}
& u_{c}^{\prime}\left(c_{t}\right)=\beta E\left[\left(f_{k}^{\prime}\left(k_{t+1}, z_{t+1}\right)+1-\delta\right) u_{c}^{\prime}\left(c_{t+1}\right)\right] \\
& k_{t+1}=(1-\delta) k_{t}+i_{t}, \quad i_{t}=y_{t}-c_{t}, \\
& y_{t}=f\left(k_{t}, z_{t}\right), \quad z_{t}=g\left(z_{t-1}\right)+\epsilon_{t} .
\end{aligned}
$$

Despite its remarkable simplicity, this model is still quite general. Typically, restrictions of a much more specific nature are however introduced when adopting parametric functional forms for the unknown functions $u, f$ and $g$. Common choices correspond to utility functions of the CRRA type, production functions of the AK family, and linear dynamics for TFP shocks,

$$
\begin{gathered}
u\left(c_{t}\right) \approx u\left(c_{t} ; \theta\right)=c_{t}^{1-\theta} /(1-\theta), \quad g\left(z_{t-1}\right) \approx g\left(z_{t-1} ; \rho\right)=\rho z_{t-1} \\
f\left(k_{t}, z_{t}\right) \approx f\left(k_{t}, z_{t} ; \alpha\right)=\exp \left(z_{t}\right) A k_{t}^{\alpha} .
\end{gathered}
$$

These choices lead to the familiar system of first-order conditions given by,

$$
\begin{aligned}
& c_{t}^{-\theta}=\beta E_{t}\left[\left(\alpha z_{t+1} k_{t+1}^{\alpha-1}+(1-\delta)\right) c_{t+1}^{-\theta}\right] \\
& k_{t+1}=(1-\delta) k_{t}+\exp \left(z_{t}\right) k_{t}^{\alpha}-c_{t} \quad, \quad z_{t}=\rho z_{t-1}+\epsilon_{t} .
\end{aligned}
$$

The classes of functions defined in (3) are indexed by a single parameter and hence very restrictive (or very small in a space entropy sense; see e.g. van der Vaart and Wellner (1996)). Consider for example the space of AK functions defined as $\left\{\exp \left(z_{t}\right) A k_{t}^{\alpha}, \alpha \in\right.$ $(0,1)\}$. It is true that each element of this class of functions satisfies important basic 
requirements for a meaningful understanding of the model, namely that output be increasing in capital stock and productivity levels. It is also true that each element of this class satisfies basic economic-theoretic requirements for the existence of a steady-state (namely that the production function be concave in the capital dimension). Unfortunately, this class of functions defines also a host of other restrictions that are unnecessarily imposed and turn out to have a strong influence on the implied dynamic behavior of consumption, output, investment and capital stock. For instance, within the AK family, curvature and slope cannot be determined independently. In effect, selecting an AK production function with larger marginal productivity of capital at the steady-state, implies necessarily selecting a production function with stronger diminishing returns to scale. Actually, no two derivatives of the production function can be determined independently. As we shall see this is an unnecessary and unjustified restriction that comes at a high cost as it renders the model incapable of explaining several observed features of the business cycle.

The solution-driven DSGE model design is instead guided by the approximation theoretic properties of the employed solution method. In particular, it relies on the properties (both advantages and limitations) of the solution method to select appropriate functional forms for the structural model. For example, in the case of the perturbation solution method (see Jin and Judd (2002) and Schmitt-Grohe and Uribe (2004)), the design of the DSGE is itself based on polynomial functions. In what follows, we take the perturbation solution to be our workhorse and call the resulting solution-driven DSGE model the perturbation-driven DSGE model. This model will make extensive use of polynomial functional forms. This is justified by the realization that selected functional forms should always:

(i) satisfy economic theory restrictions that are reflected by the solution method;

(ii) satisfy statistical restrictions that are reflected by the solution method;

(iii) be identifiable from the solution method's reduced form.

Point (i) above highlights e.g. that, since the perturbation solution method only preserves the local properties of the original functions, then theoretical restrictions should only be met locally. For example, it is irrelevant to select utility functions or production functions that satisfy monotonicity or concavity constraints globally, when in fact nonlinear 
perturbation approximations are simply incapable of ever satisfying these properties globally. Recognizing this limitation of nonlinear perturbation approximations will turn out to be an advantage.

Point (ii) reflects the same principle as (i), but it applies it to the stochastic properties of the model. In particular, it points out that while selecting functional forms with uniformly bounded derivatives is often important to ensure global stability, stationarity and ergodicity of autoregressive models (see Bougerol (1991) or Pötscher and Prucha (1997)), in this context, it is simply irrelevant since the data will be simulated from a polynomial approximation that can never achieve global stability. Again, recognizing this limitation of perturbation approximations will turn out to be a practical advantage.

Finally, point (iii) highlights the natural fact that it is useless to specify nonlinear features for structural functions independently of solution methods since identification of the former depends on the nature of the latter. For example, a linear perturbation solution is simply incapable of providing information about the curvature of structural functions. ${ }^{2}$ Likewise, it would be useless to explore higher-order solution methods if all structural functions were linear.

Given (i), (ii) and (iii) above, it is thus natural to select functional forms that mimic as closely as possible the form of the approximate perturbation solution. Specifically, we shall make use of polynomials of the same order as those of the solution method and obtain polynomial forms,

$$
u\left(c_{t} ; \boldsymbol{\theta}\right)=\sum_{i=0}^{n_{u}} \theta_{i} \hat{c}_{t}^{i} \quad, \quad f\left(k_{t}, z_{t} ; \boldsymbol{\alpha}\right)=\sum_{|i|=0}^{n_{f}} \alpha_{i} \hat{k}_{t}^{i} z_{t}^{i} \quad, \quad g\left(z_{t-1} ; \boldsymbol{\rho}\right)=\sum_{i=0}^{n_{g}} \rho_{i} z_{t-1}^{i}
$$

where $\hat{c}_{t}, \hat{k}_{t}$ and $\hat{z}_{t}$ denote variables in deviations from the steady-state. ${ }^{3}$ The selected functional forms allow us to satisfy requirements (i), (ii) and (iii) above. In particular, they allow us to (a) impose local theoretical restrictions of monotonicity and concavity that are naturally preserved by the perturbation solution; (b) impose local restrictions of

\footnotetext{
${ }^{2}$ As we shall see below, the only reason why linearized DSGE models often seem to provide information about parameters associated with curvature of functions is precisely that, in restrictive functional forms, the parameters typically bind all derivatives together and hence estimates of slope are simply imposed on the curvature. This is shown to be problematic.

${ }^{3} \sum_{|i|=0}^{n_{f}}$ is written is multi-index notation.
} 
stability that are consistent with the adopted perturbation order; and (c) identify curvature parameters that are reflected in the reduced form parameters of perturbation solution. Furthermore, the functional forms in (4) are much more flexible than typical ones. For example, in the third-order truncated power series case, it is worth noting that first, second and third derivatives (and cross-derivatives) can all be set independently of each other. As pointed out above, this was impossible when making use of the classical functional forms in (3) where all derivatives are deterministically linked and fixed by a single parameter.

The adopted functional forms imply working with the following system of first order conditions,

$$
\begin{aligned}
& \sum_{i=1}^{n_{u}} i \theta_{i} \hat{c}_{t}^{i-1}=\beta E_{t}\left[\left(\sum_{i=1}^{n_{k}} \sum_{j=0}^{n_{z}} i \alpha_{i, j} \hat{k}_{t+1}^{i-1} z_{t+1}^{j}+1-\delta\right) \sum_{i=1}^{n_{u}} i \theta_{i} \hat{c}_{t+1}^{i-1}\right] \\
& k_{t+1}=(1-\delta) k_{t}+\sum_{i=0}^{n_{k}} \sum_{j=0}^{n_{z}} \alpha_{i, j} \hat{k}_{t}^{i} z_{t}^{j}-c_{t} \quad, \quad z_{t}=\sum_{i=0}^{n_{g}} \rho_{i} z_{t-1}^{i}+\epsilon_{t}
\end{aligned}
$$

In the remainder of this paper we describe the indirect inference estimation of the perturbation-driven DSGE model and provide evidence of the importance of the perturbationdriven specification of functional forms.

\section{Data And Estimation}

Estimation of the perturbation-driven DSGE model will be based on indirect inference estimator of Gourieroux et al. (1993). The II estimator minimizes a divergences between a vector of auxiliary statistics obtained from both observed and simulated data. The II estimator of is thus defined as,

$$
\hat{\boldsymbol{\theta}}_{T}:=\arg \min _{\boldsymbol{\theta} \in \Theta_{T}}\left\|\hat{\boldsymbol{\beta}}_{T}\left(\boldsymbol{\theta}_{0}\right)-\tilde{\boldsymbol{\beta}}_{T, S}(\boldsymbol{\theta})\right\|
$$

where $\Theta$ is the parameter space of structural parameters and $\|\cdot\|$ is the employed divergence between the vector of auxiliary statistics $\hat{\boldsymbol{\beta}}_{T}\left(\boldsymbol{\theta}_{0}\right)$ that describes observed data and the vector $\tilde{\boldsymbol{\beta}}_{T, S}(\boldsymbol{\theta})$ that describes simulated data generated from the model under $\boldsymbol{\theta}$ by averaging over $S$ simulations $\tilde{\boldsymbol{\beta}}_{T, S}(\boldsymbol{\theta})=1 / S \sum_{s=1}^{S} \tilde{\boldsymbol{\beta}}_{T, s}(\boldsymbol{\theta})$. The II estimator relies on the convergence

of the auxiliary statistics $\hat{\boldsymbol{\beta}}_{T}\left(\boldsymbol{\theta}_{0}\right)$ and $\tilde{\boldsymbol{\beta}}_{T, S}(\boldsymbol{\theta})$ to limits $\boldsymbol{\beta}\left(\boldsymbol{\theta}_{0}\right)$ and $\boldsymbol{\beta}(\boldsymbol{\theta})$ as $T \rightarrow \infty$, and 
on the injective nature of the limit map so that $\boldsymbol{\beta}\left(\boldsymbol{\theta}_{0}\right) \neq \boldsymbol{\beta}(\boldsymbol{\theta}) \forall \boldsymbol{\theta} \neq \boldsymbol{\theta}_{0} \in \Theta$. Under certain regularity conditions the II estimator will then converge in probability to the unique minimizer of the limit criterion $\left\|\boldsymbol{\beta}\left(\boldsymbol{\theta}_{0}\right)-\boldsymbol{\beta}(\boldsymbol{\theta})\right\|$. Under the influence of an axiom of correct specification this limit is the true parameter $\boldsymbol{\theta}_{0}$. When the model is misspecified, i.e. $\boldsymbol{\theta}_{0} \notin \Theta$, the II estimator is consistent to a pseudo-true parameter $\boldsymbol{\theta}_{0}^{*} \in \Theta$ that provides the best approximation to the data generating process, as judged by the limit divergence $\|\cdot\|$ between the limit auxiliary vectors $\boldsymbol{\beta}\left(\boldsymbol{\theta}_{0}\right)$ and $\boldsymbol{\beta}(\boldsymbol{\theta})$. As we shall see in the following section, the perturbation-driven DSGE specification estimated using the II estimator can, at the very minimum, provide a considerably better approximation to the data generating process.

The data used in the empirical exercise that follows comprises quarterly observations of US GDP, aggregate consumption and aggregate investment in volumes from 1947 to 2012 obtained from the Federal Reserve of Saint Louis. Figure 1 plots the log of these variables in levels and the business cycle extracted by HP-filtering.
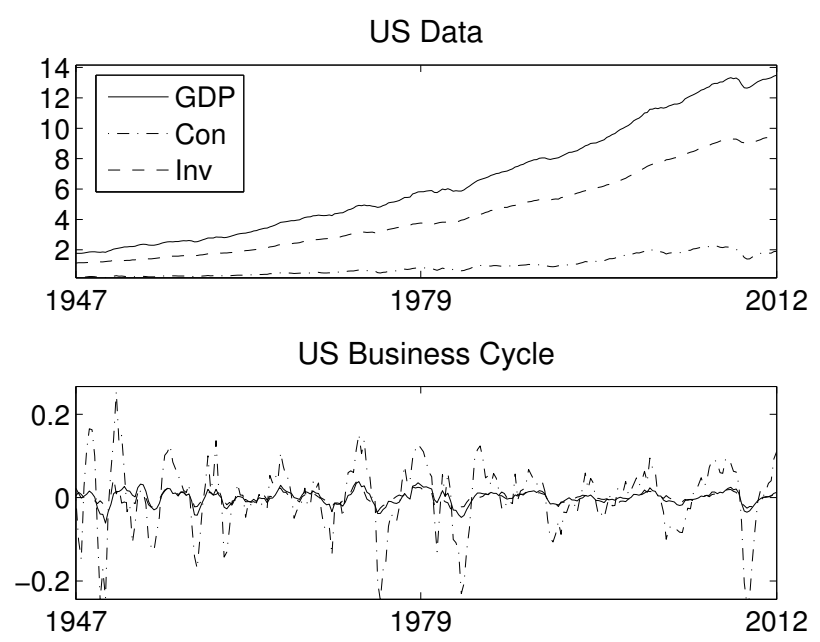

Figure 1: Logarithms of real aggregates (above) and the business cycle (below) as described by deviations of the HP-filtered logarithms (approximately \% deviations) from long run HP trend.

Table 1 reports the estimated variance ratios and autocorrelation structure of the US business cycle. The elements of this table shall constitute the vector of auxiliary statistics $\hat{\boldsymbol{\beta}}_{T}\left(\boldsymbol{\theta}_{0}\right)$ that describes observed data. Table 1 shows various well-known stylized facts of 
the business cycle. Namely, that private investment is much more volatile throughout the business cycle than GDP, and that aggregate consumption is the less volatile component. It is also interesting to point out that investment has considerably higher contemporaneous correlation with GDP than consumption, and that the contemporaneous correlation between investment and consumption is relatively weaker than any other. Finally, GDP reveals the highest values of autocorrelation, and investment the lowest. In the following section, we compare various formulations of our prototypical DSGE model in their ability to generate paths of simulated data whose properties resemble those of observed data as described by Table 1.

Auto-Covariance Structure

\begin{tabular}{cccccccc}
\hline \hline & $\sigma / \sigma_{y}$ & $r_{y}$ & $r_{c}$ & $r_{i}$ & $r_{y_{-1}}$ & $r_{c_{-1}}$ & $r_{i_{-1}}$ \\
\hline$y_{t}$ & 1.00 & - & 0.78 & 0.85 & 0.85 & 0.77 & 0.75 \\
$c_{t}$ & 0.77 & 0.78 & - & 0.70 & 0.61 & 0.82 & 0.61 \\
$i_{t}$ & 4.90 & 0.85 & 0.70 & - & 0.66 & 0.73 & 0.79 \\
\hline \hline
\end{tabular}

Table 1: US business cycle characterization. $\sigma / \sigma_{y}$ shows ratio of standard deviations w.r.t. output. $r_{x}$ shows estimated correlation with variable $x . r_{x_{-1}}$ shows estimated correlation with the lag of $x$.

Since the estimated moments reported in Table 1 will constitute the set of auxiliary statistics used for II estimation, the II estimator will be equivalent to a simulated method of moments estimator. Small sample properties of such estimators in the present context of nonlinear DSGE models are studied in Ruge-Murcia (2012).

\section{Model Analysis And Comparison}

Given the small sample size of $T=261$ typical of macroeconomic datasets, we adopt loworder truncations for the perturbation solution and power-series functions in (2) of $n_{u}=3$, $n_{k}=2, n_{z}=2, n_{g}=1$. The first-order conditions for the perturbation-driven DSGE model are thus given by,

$$
\begin{aligned}
& \theta_{1}+2 \theta_{2} \hat{c}_{t}+3 \theta_{3} \hat{c}_{t}^{2}=\beta E_{t}\left[\left(\alpha_{1}+2 \alpha_{2} \hat{k}+\alpha_{5} \hat{z}_{t}+1-\delta\right)\left(\theta_{1}+2 \theta_{2} \hat{c}_{t}+3 \theta_{3} \hat{c}_{t}^{2}\right)\right] \\
& k_{t+1}=(1-\delta) k_{t}+\alpha_{0}+\alpha_{1} \hat{k}_{t}+\alpha_{2} \hat{k}^{2}+\alpha_{3} z_{t}+\alpha_{4} z_{t}^{2}+\alpha_{5} \hat{k}_{t} z_{t}-c_{t} \quad, \quad z_{t}=\rho_{i} z_{t-1}+\epsilon_{t} .
\end{aligned}
$$


It is important to note that the first-order conditions in (5) are compatible with the increasingly popular second-order perturbation solutions. In other words, second-order perturbation solutions contain information about all the polynomial function parameters $\left(\theta_{1}, \theta_{2}, \theta_{3}, \alpha_{1}, \alpha_{2}, \alpha_{3}, \alpha_{4}, \alpha_{5},\right)$ in (5).

Table 2 presents the calibrated and estimated parameters for the standard formulation of the DSGE model postulated in (2) using both linear and quadratic solutions. Table 3 provides estimates of the parameters of the perturbation-driven DSGE (PD-DSGE) model in (5). Both models have been estimated with $\delta$ and $\beta$ at calibrated values $\delta=0.05$ and $\beta=0.987$. These values are common in the literature. Both tables report also the bounds that have been imposed on the parameter space.

\begin{tabular}{cccccc}
\hline \hline & \multirow{2}{*}{ BM } & \multirow{2}{*}{ Lin P } & \multirow{2}{*}{ Quad P } & \multicolumn{2}{c}{ Bounds } \\
& & & & $\min$ & $\max$ \\
\hline$\alpha$ & 0.25 & 0.17 & 0.17 & 0.15 & 0.35 \\
$\theta$ & 2.00 & 2.47 & 2.47 & 1.20 & 2.80 \\
$\rho$ & 0.85 & 0.89 & 0.89 & 0.73 & 0.97 \\
$\sigma$ & 0.014 & 0.013 & 0.013 & 0.010 & 0.018 \\
$\delta$ & 0.05 & - & - & - & - \\
$\beta$ & 0.987 & - & - & - & - \\
\hline \hline
\end{tabular}

Table 2: Column 1 (BM) shows benchmark calibrated parameter values $\alpha \theta, \rho, \sigma, \delta$ and $\beta$. Columns 2 and 3 (LIN P and Quad P) show parameter estimates of $\alpha \theta, \rho$ and $\sigma$ (note that $\delta$ and $\beta$ are kept at calibrated values) for the linear and quadratic perturbation solutions of the standard DSGE model design defined in (1), (2) and (3). Last two columns contain the parameter space bounds imposed during estimation and show that there were no corner solutions in the estimation algorithm.

An interesting feature of Table 2 is that the estimates of linear and quadratic model are very similar. In fact, differences are of magnitude smaller than 0.01 leaving the rounded values in the table undistinguishable. One might be tempted to interpret these results (and also the marginal differences in fit shown in Table 5) as meaning that nonlinear approximations are unnecessary in DSGE models. As we shall see however, this phenomenon is explained precisely by the incorrect functional forms being imposed. Important gains in fit and differences in parameter estimates will be obtained from nonlinear solutions when functional forms are modeled appropriately. 
Table 3 shows parameter estimates obtained under the perturbation-driven DSGE model design with quadratic perturbation solution. The auxiliary statistics and criterion function is exactly the same as that employed in the estimation of the DSGE models in Table 2.

\begin{tabular}{ccccc}
\hline \hline & \multicolumn{2}{c}{ PD-DSGE } & \multicolumn{2}{c}{ Bounds } \\
& Calibrated & Estimated & Min & Max \\
\hline$\alpha_{0}$ & 25.3 & 28.4 & 20.2 & 30.3 \\
$10 \alpha_{1}$ & 0.63 & 0.37 & 0.25 & 1.01 \\
$10^{3} \alpha_{2}$ & -0.24 & -0.25 & -0.05 & -0.43 \\
$\alpha_{3}$ & 25.3 & 36.0 & 5.1 & 45.5 \\
$\alpha_{4}$ & 12.6 & 7.23 & 2.5 & 22.7 \\
$10 \alpha_{5}$ & 0.63 & 0.26 & 0.1 & 1.1 \\
$10^{3} \theta_{1}$ & 2.43 & 3.72 & 0.2 & 4.6 \\
$10^{5} \theta_{2}$ & -12.0 & -8.36 & -1.2 & -22.8 \\
$10^{6} \theta_{3}$ & 5.93 & 2.39 & 0.6 & 11.3 \\
$\rho$ & 0.85 & 0.92 & 0.75 & 0.95 \\
$\sigma$ & 0.014 & 0.009 & 0.007 & 0.021 \\
$\delta$ & 0.05 & - & - & - \\
$\beta$ & 0.987 & - & - & - \\
\hline \hline
\end{tabular}

Table 3: Calibrated and estimated parameter values (first and second columns respectively) for perturbation-driven DSGE model defined in (1), (2) and (4). Parameters $\delta$ and $\beta$ are calibrated at benchmark calibration $\delta=0.05$ and $\beta=0.987$. Last two columns contain the parameter space bounds imposed during estimation and show that there were no corner solutions in the estimation algorithm.

Regardless of the similarity of the estimated parameters for the linear and quadratic models, it is important to note that the implied moments are quite different. Table 4 compares the observed moments estimated from the US data to those moments implied by the estimated DSGE model under linear and quadratic perturbation as well as those implied by the PD-DSGE model. The implied moments were obtained from a simulated path of $100^{5}$ periods that brings the estimation error to under 0.01 .

Table 4 reveals that increasing the solution order when imposing restrictive incorrect functional forms does not help improve the ability of the model to describe the stylized facts of the business cycle. In fact, the table shows that higher order solutions may actually harm moment fit in some occasions. For example, the estimated quadratic model implies a variance for investment that is $16 \%$ lower than the observed value of 0.687 , when the linear model actually provided an almost perfect match of this moment. This is reflected also in 


\begin{tabular}{cccccccc}
\hline \hline & Data & \multicolumn{2}{c}{ Lin P } & \multicolumn{2}{c}{ Quad P } & \multicolumn{2}{c}{ PD-DSGE } \\
& value & value & \% diff & value & \% diff & value & \% diff \\
\hline $100 \boldsymbol{\sigma}_{\mathbf{y}}$ & 2.86 & 2.97 & 4 & 2.94 & 3 & 2.87 & 0 \\
$100 \boldsymbol{\sigma}_{\mathbf{i}}$ & 68.7 & 68.6 & 0 & 57.7 & -16 & 68.4 & 0 \\
$100 \boldsymbol{\sigma}_{\mathbf{c}}$ & 1.68 & 0.63 & -62 & 0.61 & -64 & 0.99 & -41 \\
$\boldsymbol{\sigma}_{\mathbf{y}} / \boldsymbol{\sigma}_{\mathbf{i}}$ & 4.90 & 4.81 & -4 & 4.43 & -18 & 4.88 & -1 \\
$\boldsymbol{\sigma}_{\mathbf{y}} / \boldsymbol{\sigma}_{\mathbf{c}}$ & 0.77 & 0.46 & -64 & 0.46 & -65 & 0.59 & -41 \\
$\mathbf{r}_{\mathbf{y i}}$ & 0.85 & 0.98 & 15 & 0.98 & 16 & 0.97 & 14 \\
$\mathbf{r}_{\mathbf{y c}}$ & 0.78 & 0.95 & 21 & 0.95 & 21 & 0.97 & 24 \\
$\mathbf{r}_{\mathbf{i c}}$ & 0.70 & 0.86 & 24 & 0.87 & 25 & 0.88 & 27 \\
$\mathbf{r}_{\mathbf{y y} \mathbf{1}}$ & 0.85 & 0.71 & -16 & 0.72 & -15 & 0.72 & -15 \\
$\mathbf{r}_{\mathbf{y i}}$ & 0.75 & 0.74 & -3 & 0.74 & -2 & 0.73 & -3 \\
$\mathbf{r}_{\mathbf{y} \mathbf{c}_{-\mathbf{1}}}$ & 0.77 & 0.62 & -19 & 0.62 & -19 & 0.66 & -14 \\
$\mathbf{r}_{\mathbf{i y}}$ & 0.66 & 0.63 & -5 & 0.63 & -5 & 0.60 & -10 \\
$\mathbf{r}_{\mathbf{i i}}$ & 0.79 & 0.69 & -13 & 0.69 & -13 & 0.67 & -16 \\
$\mathbf{r}_{\mathbf{i c}}$ & 0.72 & 0.49 & -32 & 0.48 & -33 & 0.49 & -31 \\
$\mathbf{r}_{\mathbf{c y}}$ & 0.61 & 0.79 & 30 & 0.79 & 30 & 0.79 & 30 \\
$\mathbf{r}_{\mathbf{c i}} \mathbf{y}_{-\mathbf{1}}$ & 0.61 & 0.75 & 24 & 0.75 & 23 & 0.75 & 24 \\
$\mathbf{r}_{\mathbf{c c}}$ & 0.82 & 0.79 & -3 & 0.79 & -3 & 0.78 & -4 \\
\hline \hline
\end{tabular}

Table 4: Moments estimated from observed data (in Data column) and model implied moments obtained from simulated path of $10^{5}$ periods generated at estimated parameter values for Lin P and Quad P models in Tables 2 and PD-DSGE model in Table 3 (with values and percentage differences from data moments in 'value' and '\% diff' columns respectively). US business cycle characterization. $\sigma_{x}$ denotes simulated standard deviations of variables $x . \sigma_{y} / \sigma_{x}$ shows ratio of standard deviations w.r.t. output. $r_{x x^{\prime}}$ shows simulated unconditional correlations between variables $x$ and $x^{\prime} . r_{x x_{-1}^{\prime}}$ shows simulated unconditional correlations between $x$ and lag of $x^{\prime}$.

the implied output-to-investment variance ratio $\sigma_{y} / \sigma_{i}$ that was underestimated by the linear model in only $4 \%$ and is underestimated by $18 \%$ in the quadratic model. In the remaining moments there are marginal differences between the linear and the quadratic model. This does not happen however with the PD-DSGE model using the same quadratic solution. Indeed, by not imposing restrictive functional forms the perturbation-driven DSGE specification is capable of bringing the fit to investment variance back to an almost perfect match and to actually improve on the estimated GDP-to-investment variance ratio. Furthermore, the perturbation-driven DSGE specification is also capable of matching the variance of output almost perfectly and to improve the fit to the variance of consumption by more than $20 \%$ compared to both the linear and quadratic DSGE models. This is naturally reflected also on the improvement of the fit to the GDP-to-consumption variance ratio $\sigma_{y} / \sigma_{c}$. A very 
significant improvement can be found in the fit of the cross-autocorrelation between output and consumption $r_{y c_{-1}}$. Most of the remaining moments have only marginal differences between the standard DSGE models and the perturbation-driven DSGE specification with the exception of the cross-autocorrelations $r_{i y_{-1}}$ and $r_{i i_{-1}}$ for which the perturbation-driven DSGE specification performs worse.

Table 5 summarizes the model fit by (i) evaluating the realized criterion function of the II estimator at the estimated parameters; and (ii) providing the unweighted total difference between observed moments and simulated moments.

\begin{tabular}{ccccc}
\multicolumn{5}{c}{ Criterion Function Fit } \\
\hline \hline BM-Lin & BM-Quad & Lin P & Quad P & PD-DSGE \\
\hline 14685 & 16224 & 4521 & 4523 & 3612 \\
4.07 & 4.49 & 1.25 & 1.25 & 1.00 \\
\hline \hline \multicolumn{5}{c}{ Total Moment Difference } \\
\hline \hline BM-Lin & BM-Quad & Lin P & Quad P & PD-DSGE \\
\hline 4.95 & 5.06 & 3.38 & 3.71 & 2.97 \\
1.67 & 1.71 & 1.14 & 1.25 & 1.00 \\
\hline \hline
\end{tabular}

Table 5: The BM-Lin and BM-Quad columns report values for the benchmark calibrated model with linear and quadratic perturbation solutions, respectively. The Lin Pert and Quad Pert columns report values for the estimated benchmark model under linear and quadratic perturbation solutions, respectively. Finally, the perturbation-driven specification column reports the value for the estimated PD-DSGE model. The criterion fit uses optimal weighting matrix and is hence weighted by uncertainty in estimates. Total moment difference is unweighted sum of percentage differences. Second rows report standardized values w.r.t. to the PD-DSGE model.

In Table 5 it is clear that model estimation improves the fit on any model. Indeed, estimated models reveal a great improvement in both criterion fit and a total moment difference when compared to the calibrated model which was used as a starting point for the indirect inference estimation of all models. Most importantly, the calibrated model solved with quadratic solution (BM-Quad) actually does a worse job in describing the observed moments than the calibrated model with linear solution. This is evidence once again, that imposing incorrect functional forms on the data is harmful. Similar evidence is found when comparing the estimated linear and quadratic models. While the criterion function seems 
relatively unchanged, the unweighted total moment difference reveals that the quadratic model provides a worse fit than the linear model. Again, it is important to highlight that it would be a mistake to interpret these results as meaning that nonlinear approximations are unnecessary in DSGE models. On the contrary, this phenomenon is explained precisely by the incorrect functional forms being imposed. This is supported by the fact that the PD-DSGE model provides a remarkable improvement in fit, both in criterion function terms and total moment difference. In effect, the criterion fit is $25 \%$ better than both the linear and quadratic estimated DSGE models, and total moment difference is $14 \%$ better than any alternative.

The difficulty of restrictive DSGE models in improving model fit with higher-order solutions can be well understood by looking at the curvature properties of the estimated utility and production functions. Table 6 reports both the calibrated and estimated first, second and third derivatives at the steady-state of the utility function under the standard model and PD-DSGE model. The estimated utility functions and respective derivatives are plotted in Figure 3.

\begin{tabular}{cccc}
\multicolumn{4}{c}{ Estimated Utility Function Derivatives } \\
\hline \hline Model & $10^{3} \mathbf{u}_{\mathbf{c}}$ & $10^{5} \mathbf{u}_{\mathbf{c c}}$ & $\mathbf{u}_{\mathbf{c c c}}$ \\
\hline $\mathbf{B M}$ & 6.00 & -93.0 & 216.1 \\
Lin/Quad P & 1.82 & -34.8 & 93.4 \\
PD-DSGE & 3.72 & -16.7 & 14.3 \\
\hline \hline
\end{tabular}

Table 6: First $\left(\mathbf{u}_{\mathbf{c}}\right)$, second $\left(\mathbf{u}_{\mathbf{c c}}\right)$ and third $\left(\mathbf{u}_{c c c}\right)$ derivatives of the utility function evaluated at the steady-state for the benchmark calibrated model (BM), the linear and quadratic perturbation (Lin/Quad P) solutions of the standard DSGE model design defined in (1), (2) and (3), and the perturbation-driven DSGE (PD-DSGE).

Clearly, the benchmark DSGE model calibrated at $\theta=2$ implied a first and third derivative that were too large and a second derivative that was too small. This appreciation is supported by the fact that all estimated models diverge from the Benchmark in a consistent way in terms of first second and third derivatives. Unfortunately in the CRRA utility function these derivatives can not be set independently and as a result the estimated value of $\theta$ must 'compromise' between slope and curvature. For example, increasing the slope of the CRRA utility function implies necessarily reducing its curvature (second derivative 
rises closer to zero and the utility become flatter); see Figure 2 which plots the derivatives of the CRAA utility function as a function of $\theta$.
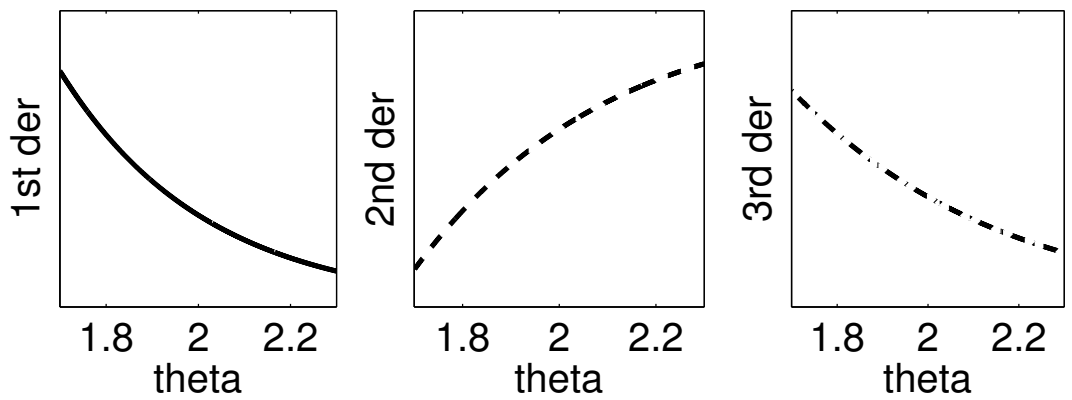

Figure 2: Plots of CRRA utility derivatives as functions of the parameter $\theta$ that shows unique relation between derivatives in CRRA utility function. An increase in $\theta$ implies a decrease in slope and an increase in curvature.
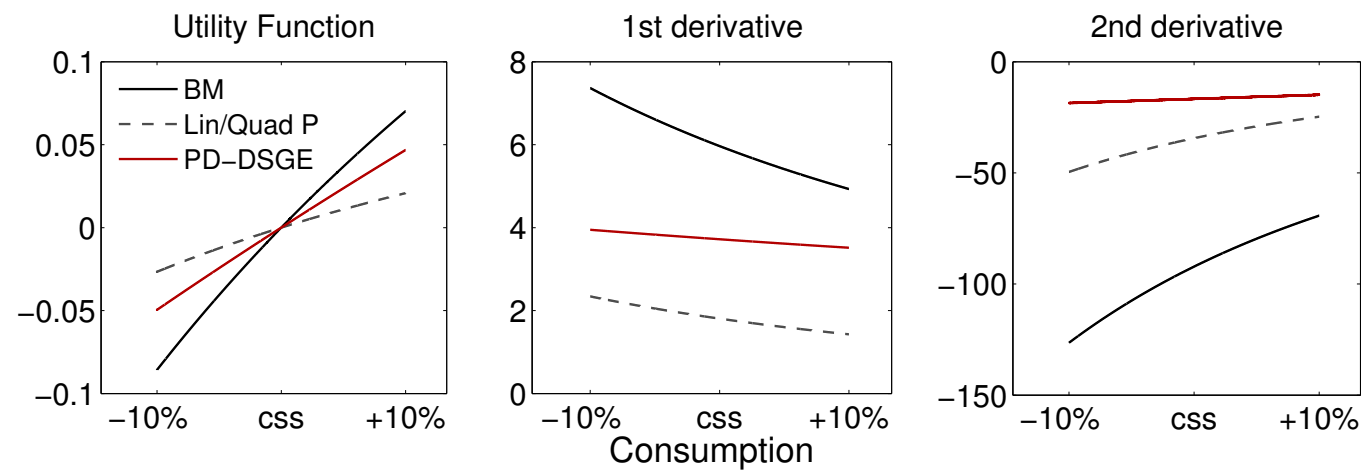

Figure 3: Plots of utility functions (left), first derivatives (center) and second derivatives (right) over $10 \%$ deviations of consumption from its steady-state value and under calibrated benchmark model (BM), linear and quadratic estimated models (Lin/Quad P) and perturbation-driven DSGE (PD-DSGE).

The effect of the restriction characterized in Figure 2 is clear. Figure 3 shows that, compared to the perturbation-driven specification utility function whose slope and curvature are estimated freely, the estimated CRRA utility must set the slope of the utility function much lower in order to reduce the curvature of the function (i.e. in order to bring the second derivative close to zero). The left graph in Figure 3 shows precisely the effects of such restrictions on estimated utility functions. In the CRRA utility function, a smaller $\theta$ would increase the slope of the utility function, but also, at the same time, increase the curvature (i.e. pull the second derivative further down) which is already higher than 
the freely estimated one in the perturbation-driven specification utility. This restriction compromises the ability of the standard DSGE to fit the data appropriately.

Similar analysis can be made about the estimated production function. In particular, Table 7 suggests that the calibrated AK production function implies a slope for the production function that is too large and a curvature that is too small.

\begin{tabular}{cccccc}
\multicolumn{6}{c}{ Estimated Production Function } \\
\hline \hline & $\mathbf{1 0 f}_{\mathbf{k}}$ & $\mathbf{1 0}^{\mathbf{3}} \mathbf{f}_{\mathbf{k k}}$ & $\mathbf{f}_{\mathbf{z}}$ & $\mathbf{f}_{\mathbf{z z}}$ & $\mathbf{1 0 f}_{\mathbf{k z}}$ \\
\hline BM & 0.63 & -0.47 & 25.3 & 25.3 & 0.63 \\
Lin/Quad P & 0.29 & -0.24 & 17.5 & 17.5 & 0.29 \\
PD-DSGE & 0.37 & -0.49 & 36.0 & 14.5 & 0.26 \\
\hline \hline
\end{tabular}

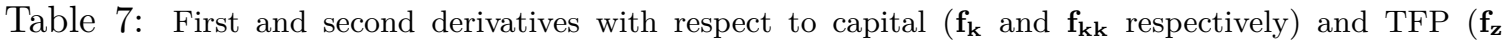
and $\mathbf{f}_{\mathbf{z z}}$ respectively) and cross-derivative $\left(\mathbf{f}_{\mathbf{k z}}\right)$ of the production function evaluated at steady-state under calibrated benchmark model (BM), linear and quadratic estimated models (Lin/Quad P) and perturbationdriven DSGE (PD-DSGE).

In effect, the freely estimated derivatives of the perturbation-driven specification production function suggest that while the slope of the production function could be reduced, its curvature should be stronger. However, in the AK family of production functions, a smaller estimate of $\alpha$, besides reducing the slope of the production, would simultaneously imply a reduction in curvature through an increase in the second derivative (bringing it closer to zero) and making the production function flatter. Figure 4 plots the derivatives of the AK production function as functions of $\alpha$.
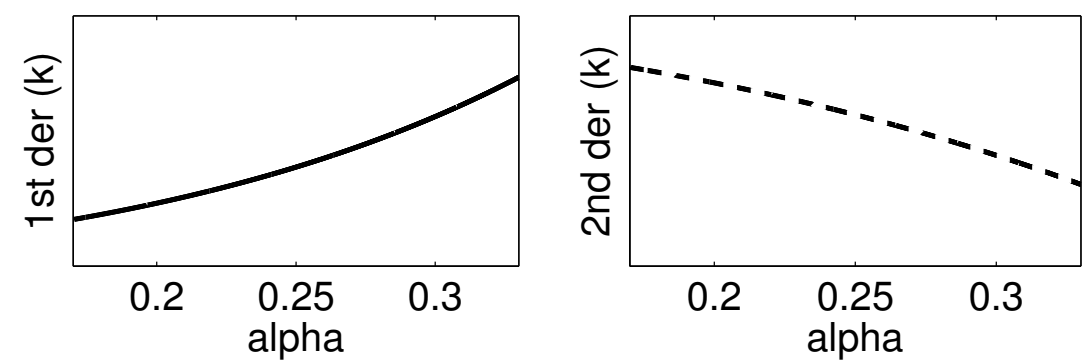

Figure 4: First (left) and second (right) derivatives of AK production w.r.t. capital as functions of parameter $\alpha$.

Figure 5 plots the isoquants of the calibrated and estimated production functions. Interestingly, the freely estimated perturbation-driven specification production function also 
provides an estimated production function that is convex in the direction of $z_{t}$. However, the convexity is not of the exponential type (as postulated by the AK production function). On the contrary, first and second derivative on the direction of $z_{t}$ are quite different. In particular, the first derivative is much larger than the second, yielding the production function with a larger slope but less pronounced curvature than the functional form implied by the estimated AK function.
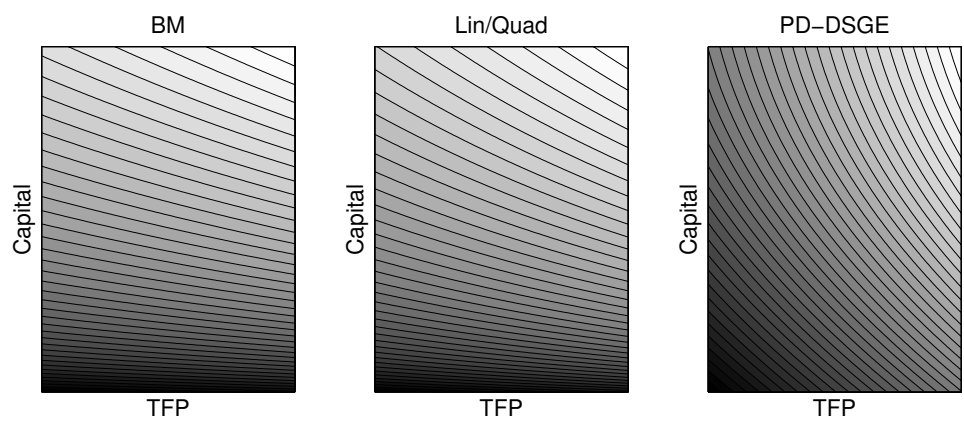

Figure 5: Contour line of production function under calibrated benchmark model (left), linear and quadratic estimated standard DSGE model (center) and estimated perturbationdriven DSGE model (right).

Finally, Table 8 reveals fundamental differences in the estimates of various economic quantities of interest obtained from both the standard DSGE and PD-DSGE models. In particular, Table 8 provides estimates of the Arrow-Pratt coefficient of relative risk aversion (APC), elasticity of intertemporal substitution (IES), marginal productivity of capital $(\mathrm{MPK})$, and relative curvature of the production function in the capital dimension (RCK), TFP dimension (RCZ) and cross dimension (RCKZ).

\begin{tabular}{ccccccc}
\hline \hline & APC & EIS & MPK & RCK & RCZ & RCKZ \\
\hline Calibrated & 2.00 & 0.50 & 0.63 & -0.75 & 1.00 & 0.040 \\
Lin/Quad P & 2.47 & 0.41 & 0.30 & -0.83 & 1.00 & 0.057 \\
PD-DSGE & 0.91 & 1.10 & 0.37 & -1.33 & 0.40 & 0.020 \\
\hline \hline
\end{tabular}

Table 8: Steady-state values of relevant economic quantities estimated under different model formulations. Relative curvatures defined as ratio of second and first derivatives.

It is important to highlight that these quantities can be easily obtained regardless of 
the adopted functional forms. Consider for example the APC which is defined as,

$$
\operatorname{APC}(c)=-\frac{u^{\prime \prime}(c)}{u^{\prime}(c)}
$$

In general, this coefficient is a function of the first two derivatives of the utility function $u^{\prime}$ and $u^{\prime \prime}$ and the consumption level $c$. In the special case of the CRRA utility function the APC is constant in $c$ and given by $A P C(c)=\theta \forall c$. However, regardless of the form of the utility function, the APC is defined as long as the first and second derivatives of the utility function are well defined. Unfortunately, as we have seen before, the estimated derivatives of the utility function can be severely biased if the utility function is too restrictive. Table 6 showed that estimating correctly $u^{\prime}$ and $u^{\prime \prime}$ in the CRRA class can be complicated when both derivatives depend on the same parameter $\theta$. Here, Table 8 reveals that the flexible estimation of these derivatives offered by the perturbation-driven DSGE specification implies a much lower coefficient at the steady-state than that implied by the CRRA utility. This is naturally reflected in a larger estimate for the EIS under the perturbation-driven DSGE specification than that obtained under the standard DSGE formulation.

Finally, we note that these different estimates have important policy implications. For example, the estimated EIS below unity in the CRRA utility function implies that, given rise of interest rates, the income effect dominates the inter-temporal substitution effect. This implies that consumption increases as a result of a rise in interest rates because the effect of a rise in interest in creating a more favorable intertemporal budget constraint is larger than the substitution effect that makes savings relatively more attractive under a higher interest. A logarithmic utility function implies a $E I S=1$ and hence that both effects cancel out. The perturbation-driven specification utility function where both first and second derivatives are freely estimated lead to an EIS above unity. This implies that the substitution effect dominates and consumption drops in response to a rise in interest rates as consumers shift their budget towards savings. This example shows the dramatic change to the model's policy implications that can occur as a result of appropriately modeling functional forms. 


\section{Conclusion}

This paper proposed a novel DSGE model design strategy based on adopting functional forms that are consistent with the solution methods employed and highlighted the limitations of DSGE model design with restrictive functional forms. The enhanced flexibility of the solution-driven DSGE specification was shown to significantly improve the fit of the prototypical DSGE structure and deliver very different estimates of function shapes and implied coefficients of risk aversion, elasticity of substitution, marginal capital productivity and others.

The paper's main objective was that of introducing the solution-driven DSGE specification, explaining its main features and using a prototypical DSGE to analyze some of its potential strengths. Future research will be devoted to the application of the solution-driven specification in larger DSGE models. In effect, the larger the model, the more likely it is for the solution-driven specification design to have a significant influence on fit. This is so for three reasons. First, larger models contain more equations with functions whose form is unknown, and hence, a more significant role can be played by the solution-driven specification design strategy. Second, the extra data allows for higher order approximations, especially when focusing on approximating a small number of functions. Third, DSGE models with more variables have much larger variance-covariance matrices (whose number of elements grow exponentially with each new variable), but typically only few new parameters. Hence, it becomes comparatively more difficult to find a good fit to all moments with few extra parameters. As this paper revealed, this is precisely where the solution-driven specification design gains advantage over traditional model design.

\section{REFERENCES}

Aruoba, S., Fernandez-Villaverde, J., and Rubio-Ramirez, F. (2006). Comparing solution methods for dynamic equilibrium economies. Journal of Economic Dynamics and Control, 30(12):24772508 .

Blanchard, O. and Kahn, C. M. (1980). The solution of linear difference models under rational expectations. Econometrica, 48(5):1305-11.

Bougerol, P. (1991). Kalman Filtering with Random Coefficients and Contractions. Prépublications de l'Institut Elie Cartan. Univ. de Nancy.

Diewert, W. E. and Wales, T. J. (1987). Flexible functional forms and global curvature conditions. Econometrica, 55(1):43-68. 
Fernandez-Villaverde, J. and Rubio-Ramirez, J. F. (2006). Solving dsge models with perturbation methods and a change of variables. Journal of Economic Dynamics and Control, 30(12):25092531 .

Gourieroux, C., Monfort, A., and Renault, E. (1993). Indirect inference. Journal of Applied Econometrics, 8:85-118.

Granger, C. W. J. and Teräsvirta, T. (1993). Modelling Non-Linear Economic Relationships. Oxford University Press.

Jin, H. and Judd, K. (2002). Perturbation methods for general dynamic stochastic models. Mimeo, Hoover Institution.

Judd, K. (1992). Projection methods for solving aggregate growth models. Journal of Economic Theory, 58(2):410-452.

Judd, K. (1998). Numerical methods in economics. Cambridge, Mass: MIT Press.

Kim, J. and Ruge-Murcia, F. J. (2009). How much inflation is necessary to grease the wheels? Journal of Monetary Economics, 56(3):365-377.

Lau, L. (1986). Functional forms in econometrics model building. In Griliches, Z. and Intriligator, M. D., editors, Handbook of Econometrics, volume III. Elsevire Science Publishers.

Pötscher, B. M. and Prucha, I. R. (1997). Dynamic Nonlinear Econometric Models: Asymptotic Theory. Springer-Verlag.

Ruge-Murcia, F. (2012). Estimating nonlinear \{DSGE\} models by the simulated method of moments: With an application to business cycles. Journal of Economic Dynamics and Control, 36(6):914 938.

Schmitt-Grohe, S. and Uribe, M. (2004). Solving dynamic general equilibrium models using a second-order approximation to the policy function. Journal of Economic Dynamics and Control, $28(4): 755-775$.

Uribe, M. (2011). Risk matters: The real effects of volatility shocks. American Economic Review, 101(6):2530-61.

van der Vaart, A. W. and Wellner, J. A. (1996). Weak convergence and empirical processes. SpringerVerlag, New York. 